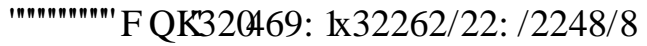

\title{
DECOMPOSITION AND ESTIMATION OF MULTI-STATE SYSTEMS BY DYNAMIC RELIABILITY INDICES
}

\author{
Zaitseva Elena, Levashenko Vitaly, Matiaško Karol \\ University of Žilina, Faculty of Management Science and Informatics \\ ul.Univerzitna 8215/1, 01026 \\ Zilina, Slovakia \\ e-mails: Elena.Zaitseva@fri.uniza.sk, Vitaly.Levashenko@fri.uniza.sk, \\ Karol.Matiasko arri.uniza.sk
}

\begin{abstract}
Some typical configurations of Multi-State System and their mathematical descriptions are considered in paper with relation to Reliability Analysis. Multiple-Valued Logic is applied for these descriptions synthesis and Dynamic Reliability Indices are used for Multi-State System reliability estimation. We concentrate on series and parallel systems, because these structures are basic for most of the technical system. We get measures of reliability for the failure and restoration of this system.
\end{abstract}

Keywords: Multi-State System, Dynamic Reliability Indices, Direct Partial Logic Derivatives, Decomposition 


\section{DECOMPOSITION AND ESTIMATION OF MULTI- STATE SYSTEMS BY DYNAMIC RELIABILITY INDICES}

\section{Introduction}

A discrete model has been used in reliability analysis frequently and well known two mathematical tapes of this model: Binary System and MultiState System (MSS). The system and its components are allowed to have only two possible states (completely failed and perfect functioning) in a Binary System. MSS reliability analysis is a more flexible approach to evaluate system reliability, as it can be used when both the system and its components may experience more than two states, to include, completely failed, partially failed, partially functioning and perfect functioning. The MSS scientific achievement has been documented in [1].

Principal measures of MSS are reliability indices that have been computed as the reliability of the specified level of the system states, or its modification depending on the change of the system component states [1 3]. There are different mathematical tools for determining these indices. One of them is structure function tool. New methodology for MSS reliability estimation by the system structure function is proposed in [4]. This methodology has been developed from angle of Multiple-Valued Logic (MVL) and Logical Differential Calculus. Dynamic Reliability Indices (DRIs) are proposed as measures of MSS reliability. These indices are used to estimate changes of system reliability caused by changes in the states of its components $[4-6]$.

DRIs are probabilistic indices and include two groups: Component Dynamic Reliability Indices (CDRIs) and Dynamic Integrated Reliability Indices (DIRIs). CDRIs allows measuring an influence of each individual component or a fixed group of components to the system reliability. DIRIs characterize a probability of impact of one or some of system components to the system reliability. These indices define the boundary states of MSS and conditions of being and changing of these states depending on the change of the system component states. 
However, these indices have high dimensionality and there are problems in their application for real-word engineering problems. Investigation and calculation of reliability indices for typical MSS can be one of the decisions of this problem [7]. Authors of papers [5, 6] develop the investigation method of MSS by DRIs and proposes measures for parallel, series and $k$-out-of- $n$ MSS and proposes a mathematical model of MSS that is allowed to present three typical systems (parallel, series and $k$-out-of- $n$ ).

In this paper we propose another decision of this problem: the algorithms of MSS decomposition are suggested. Decomposition of MSS structure function is investigated and calculation of DRIs for this system by structure function is presented in the paper. Estimation of computational complexity for DRIs determination is realized for algorithms without MSS decomposition and algorithms with MSS decomposition. The algorithm of MSS decomposition and its estimation by DRIs can be used to apply in information and communications systems.

\section{Basic Conception}

The MSS and each of $n$ components can be in one of $m$ possible states: from the complete failure (it is 0 ) to the perfect functioning (it is $m-1$ ). Every system component $x_{i}$ is characterized by probability of the performance rate:

$p_{i, s_{i}}=\operatorname{Pr}\left\{x_{i}=s_{i}\right\}, \quad s_{i}=0, \ldots, m-1$.

The system reliability (system state) depends of its components state and is defined by the structure function $[1,3,7]$ :

$\phi\left(x_{1}, \ldots, x_{n}\right)=\phi(x):\{0, \ldots, m-1\}^{n} \rightarrow\{0, \ldots, m-1\}$.

The following assumptions are used for structure functions as shown in (2) that are peculiar to reliability analysis [1-4]: $(a)$ it is the MVL function; $(b)$ the structure function is monotone and $\phi(s)=s(s \in\{0, \ldots, m-1\}) ;(c)$ all components are $s$-independent and are relevant to the system.

The assumption (a) is important to exploit the mathematical tools of MVL for the reliability analysis. Direct Partial Logic Derivatives of MVL function are proposed for reliability analysis of a MSS in [4-6]. In these papers, a Direct Partial Logic Derivative with respect to variables vector for a MSS 
structure function allows to estimate change of MSS reliability caused by state changes of some system components. These components are interpreted as vector components.

Therefore Direct Partial Logic Derivatives of a structure function $\phi(x)$ of $n$ variables with respect to vector $\boldsymbol{x}^{(p)}=\left(x_{i_{1}}, x_{i_{2}}, \ldots, x_{i_{p}}\right)$ is defined as $[4-6]$ :

$\partial \phi(j \rightarrow h) / \partial \boldsymbol{x}^{(p)}\left(\boldsymbol{a}^{(p)} \rightarrow \boldsymbol{b}^{(p)}\right)=\left\{\begin{array}{ll}m-1, & \text { if } \phi\left(\boldsymbol{a}^{(p)}, \boldsymbol{x}\right)=j \boldsymbol{\&} \phi\left(\boldsymbol{b}^{(p)}, \boldsymbol{x}\right)=h \\ 0, & \text { otherwards }\end{array}\right.$,

where $\phi\left(\boldsymbol{a}^{(\boldsymbol{p})}, \boldsymbol{x}\right)=\phi\left(a_{i_{1}}, \ldots, a_{i_{p}}, \boldsymbol{x}\right)$ is value of structure function, when $x_{i_{1}}=a_{i_{1}}, \ldots, x_{i_{p}}=a_{i_{p}}$ and $\phi\left(\boldsymbol{b}^{(\boldsymbol{p})}, \boldsymbol{x}\right)=\phi\left(b_{i_{1}}, \ldots, b_{i_{p}}, \boldsymbol{x}\right)$ is value of structure function, when $x_{i_{1}}=b_{i_{1}}, \ldots, x_{i_{p}}=b_{i_{p}}$.

Direct Partial Logic Derivative (3) with respect to variables vector for a MSS structure function permits to analyse the system reliability change from $\mathrm{j}$ to $\mathrm{h}$ when every variable values of this vector changes from $\mathrm{a}$ to $\mathrm{b}$. Even when Direct Partial Logic Derivative can always be used to analyze changes of system reliability, it is not practical for real-world systems because it is computational complex. Therefore in MSS reliability analysis the main interest has been in the most essential system reliability changes: system failure and system repair.

The MSS failure caused by a state change of one system component has been defined in paper [4] using Direct Partial Logic Derivatives terminology. This definition is developed for changes of fixed system components in papers [6].

So, the system failure is represented using Direct Partial Logic Derivative terminology as [4-6]:

$\partial \phi(1 \rightarrow 0) / \partial \boldsymbol{x}^{(p)}(1 \rightarrow 0)$,

where $\mathbf{1}=(\underbrace{1, \ldots, 1}_{p})$ and $\mathbf{0}=(\underbrace{0, \ldots, 0}_{p})$. 
The MSS repair in Direct Partial Logic Derivative terminology is declared as the structure function change from zero into $h(\phi(x): 0 \rightarrow h)$ and as $p$ failed system components simultaneous changes from zero into $(m-1)$ :

$\partial \phi(0 \rightarrow h) / \partial \boldsymbol{x}^{(p)}(0 \rightarrow(m-1))$,

where $h \in\{1, \ldots, m-1\} ; \mathbf{0}=(\underbrace{0, \ldots, 0}_{p})$ and $(\boldsymbol{m}-\mathbf{1})=(\underbrace{(m-1), \ldots,(m-1)}_{p})$.

\section{Measures of MSS Failure and Repair}

The Reliability Function, $R(j)$, is one of best known MSS reliability measures. It is probability that system reliability is great than or equal to the level $j[2-5]$ :

$R(j)=\operatorname{Pr}\{\phi(\boldsymbol{x})=. j\}, j \in(0,1, \ldots, m-1\}$.

But measures (12) do not permit the analysis of the change in system reliability that is caused by a change of component states. At the same time, in papers $[6,7]$ indices for the estimation of the influence of component states changes into the MSS reliability have been proposed. These indices are named as DRIs and defined as:

$$
\widetilde{R}=\operatorname{Pr}\left\{\partial \phi(j \rightarrow h) / \partial \boldsymbol{x}^{(p)}\left(\boldsymbol{a}^{(p)} \rightarrow \boldsymbol{b}^{(p)}\right) \neq 0\right\}
$$

Two groups of DRIs are in papers [4 - 6]. They are CDRIs and DIRIs. CDRIs are estimates of the influence of state change(s) in one or some system component(s) on the change of system reliability. DIRIs are another group of DRI's representing probabilities of system reliability changes when one or some system components states changes.

Definition 1. CDRIs of MSS failure are probabilities of MSS failure caused by breakdown of some system components [6]:

$$
P_{f}\left(\boldsymbol{x}^{(p)}\right)=\left(\rho_{f} / \rho_{1}\right) \cdot \prod_{j=1}^{p} p_{i_{j}, 1},
$$

where $\rho_{f}$ is number of system states when the breakdown of $p$ system components results the system failure (this number is calculated as numbers 
of nonzero Direct Partial Logic Derivative (4)); $\rho_{1}$ is number of system states when $\phi\left(1_{i_{1}}, \ldots, 1_{i_{p}}, \boldsymbol{x}\right)=1$ (it is computed by structure function of MSS); and $p_{i, 1}$ is probability according to (1).

Definition 2. CDRIs of a MSS repair are probabilities of MSS repair caused by replacements of $p$ failed system components [6]:

$$
P_{r}\left(\boldsymbol{x}^{(p)}\right)=\left(\sum_{h=1}^{m-1} \rho_{r}^{(h)} / \rho_{0}\right) \cdot \prod_{j=1}^{p} p_{i_{j}, 0}
$$

where $\rho_{r}^{(h)}$ is number of system states when simultaneous replacements of $p$ system components results the system repair and it is calculated by Direct Partial Logic Derivative (5); $\rho_{0}$ is number system states for which $\phi\left(0_{i_{1}}, \ldots, 0_{i_{p}}, \boldsymbol{x}\right)=0 ; p_{i_{j}, 0}$ is probability of component state according to (1).

In paper [5] CDRIs for parallel and series MSS was determined (Table 1). These expressions define the dependence of the MSS failure on breakdown of a system components and MSS repairing on the replacement of the failure components. CDRIs are calculated by the component probabilities, parameters $m$ (number of reliability levels) and $n$ (number of system components) only. Direct Partial Logic Derivates are not calculated in these cases and a complexity of CDRIs calculation reduces.

Table 1. CDRIs for parallel and series MSS

\begin{tabular}{|l|l|l|}
\hline & Parallel MSS & Series MSS \\
\hline MSS failure & $P_{f}\left(\boldsymbol{x}^{(p)}\right)=\prod_{j=1}^{p} p_{i_{j}, 1} / 2^{n-p}$ & $P_{f}\left(\boldsymbol{x}^{(p)}\right)=\prod_{j=1}^{p} p_{j_{j}, 1_{j}}$ \\
\hline MSS repair & $P_{r}\left(\boldsymbol{x}^{(p)}\right)=\prod_{j=1}^{p} p_{i_{j}, 0}$ & $P_{r}\left(\boldsymbol{x}^{(p)}\right)=\left(\frac{m-1}{m}\right)^{n-p} \cdot \prod_{j=1}^{p} p_{i_{j}, 0}$ \\
\hline
\end{tabular}

Definition 3. DIRIs for MSS failure are probabilities of MSS failure caused by any $p$ system components fail:

$$
P_{f}=\sum_{z} P_{f}\left(x^{(p)}\right) \prod_{z-1}\left(1-P_{f}\left(\bar{x}^{(p)}\right)\right)
$$


where $P_{f}\left(\boldsymbol{x}^{(p)}\right)$ is CDRIs (8); $\overline{\boldsymbol{x}}^{(\boldsymbol{p})}$ is the variable vector of $p$ variables for which $\bar{x}^{(p)} \neq \boldsymbol{x}^{(p)} ; z$ is number of combinations of $n$ things taken $p$.

Definition 4. DIRIs for MSS repair are probabilities of MSS repair caused by replacements of any $\boldsymbol{p}$ system components:

$P_{r}=\sum_{z} P_{r}\left(x^{(p)}\right) \prod_{z-1}\left(1-P_{r}\left(\bar{x}^{(p)}\right)\right)$

where $P_{r}\left(\boldsymbol{x}^{(p)}\right)$ is CDRI's (9).

The assumption $(c)$ for structure function of MSS that all components are independent and relevant to the system must hold for the DIRIs definitions above.

\section{MSS Decomposition}

The structure function of MSS is interpreted as MVL function. There are well-know methods of MVL function decomposition that allow to reduce computation complexity for this function analysis. One of these methods decomposition is in Fig.1 [9]. Therefore a structure function is presented by parallel and series function for which CDRIs are in Table 1.

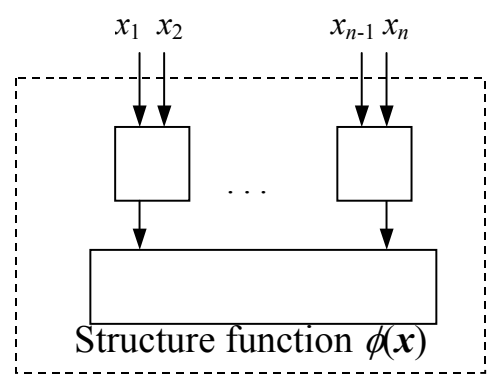

Fig. 1. Structure function decomposition

For example, consider consider 2-out-of-3 MSS with three levels of reliability $(m=3, n=3)$. The component probability of this system 
components is equal and are: $p_{i, 0}=0.1, p_{i, 1}=0.3$ and $p_{i, 2}=0.6$. In MVL terminology this function is declared as:

$\phi(\boldsymbol{x})=\mathrm{OR}\left(\operatorname{AND}\left(x_{1}, x_{2}\right), \operatorname{AND}\left(x_{1}, x_{3}\right), \operatorname{AND}\left(x_{2}, x_{3}\right)\right)$

where $\operatorname{AND}\left(x_{i}, x_{j}\right)=\min \left(x_{i}, x_{j}\right) ; \operatorname{OR}\left(x_{i}, x_{j}\right)=\max \left(x_{i}, x_{j}\right)$.

The procedure of computation of CDRIs of this system failure is in Fig.2 if the first component breakdown: $P_{f}\left(x_{1}\right)=0.025$. CDRIs for function AND and $\mathrm{OR}$ are calculated by equation form Table 1 .

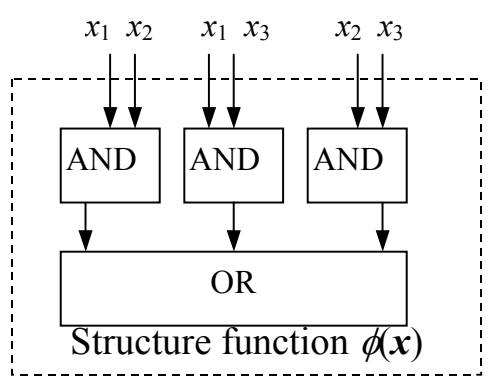

Fig. 2. Calculation CDIRs of 2-out-of-3 MSS failure

Acknowledgement This work was partially supported by grants of Scientific Grant Agency of the Ministry of Education of Slovak Republic and the Slovak Academy of Science (Vega 1/3084/06 and MVTS Bil/Fin/SR/ŽU/06).

\section{References}

1. Lisnianski A.., Levitin G.: Multi-State System Reliability. Assessment, Optimization and Applications, World scientific, 2003.

2. Xie, M. \& Dai, Y.-S. \& Poh, K.-L.: Multi-State System reliability. Computing System Reliability. Models and Analysis. Kluwer Academic Publishers, New York, 2004.

3. Shooman M.L.: Reliability of Computer Systems and Networks: Fault Tolerance, Analysis, and Design. John Wiley \& Sons, Inc, New York, 2002.

4. Zaitseva E.: Dynamic Reliability Indices for Multi-State System., Journal of dynamic system \& geometric theories, 1(2), 2003..

5. Zaitseva E., Levashenko V.: Dynamic Reliability Indices for parallel, series and k-out-of-n Multi-State System. Proc. of the IEEE 52nd Annual Reliability 
\& Maintainability Symposium (RAMS). January 23-26, Newport Beach, California, USA, 2006.

6. Zaitseva E., Puuronen S.: Estimation of Multi-State system reliability depending on changes of some system component efficiencies, Proc. of European Safety and Reliability Conference (ESREL 2007), 25-27 June, Stavanger, Norway, 2007.

7. Hung J., Zuo M.J., Multi-State $k$-out-of- $n$ System Model and its Applications, Proc Ann. Reliability \& Maintainability Symp., 2000, pp.264-268.

8. Pham, H. Handbook of Reliability Engineering. Springer, 2003.

9. Sasao T.: Switching Theory for Logic Synthesis, Kluwer Academic Publishers, Norwell, MA, 1999.

Elena Zaitseva (doc., $\mathrm{PhD}$ ) received master degree in 1989 from the Radioengeneering Institute, Minsk, Belarus and Ph.D. in Computer Science from the State University of Informatics and Radioelectronics, Minsk, Belarus in 1994. She works in Miultiple-Valued Logic, Logic Design, and Reliability Analysis. The principal area of her interests is a reliability analysis of Multi-State System. Her current research and commercialization interests are in the wide area of Design of Reliability System. She has published about 100 refereed papers in international journal and conferences.

Vitaly Levashenko (doc., $\mathrm{PhD}$ ) received master degree in 1991 from the Radioengeneering Institute, Minsk, Belarus and Ph.D. in Computer Science from the State University of Informatics and Radioelectronics, Minsk, Belarus in 1996. He works in Miultiple-Valued and Fuzzy Logic, Data Mining. He was a program committee member and vice-chair of several international conferences from 1996 to 2007 years. He has published about 60 refereed papers in international journal and conferences.

Karol Matiaško (doc., $\mathrm{PhD}$ ) received $\mathrm{PhD}$ in Technical Cybernetics in 1986 at the University of Transport and Communication in Žilina like employee of the Research Institute of Transport. His research and educational activities have been oriented in the area of Database system, Distributed processing and Data processing. K. Matiaško has published more 100 refereed papers in international journal and conferences and have been supervise of more 10 projects. 
